\title{
Effects of a Brief Meditation Training on Negative Affect, Trait Anxiety and Concentrated Attention ${ }^{1}$
}

\author{
Carolina Baptista Menezes ${ }^{2}$ \\ Universidade Federal do Rio Grande do Sul, \\ Porto Alegre-RS, Brazil
}

\author{
Lisiane Bizarro \\ Universidade Federal do Rio Grande do Sul, \\ Porto Alegre-RS, Brazil
}

\begin{abstract}
Meditation has been associated with positive psychological outcomes, but few studies have investigated brief interventions. This randomized controlled pilot study assessed the effects of five days of focused meditation on positive and negative affect, state and trait anxiety, as well as concentrated attention in a nonclinical sample distributed in two groups (experimental $=14,51.8 \%$ female, $M_{\text {age }}=23.9$; control $=19,62 \%$ female, $M_{\text {age }}=24.9$ ). The instruments used were the Positive Affect and Negative Affect Scale, State and Trait Anxiety Inventory, and the Concentrated Attention Test. The meditation group reduced negative affect and trait anxiety, and also improved correct responses on the attention test, relative to controls. These preliminary findings indicate that even short focused meditation training may help improve some psychological variables. It is discussed that the early manifestation of these benefits may be especially relevant to strengthen the motivation to continue and practice regularly.
\end{abstract}

Keywords: meditation, anxiety, affection, attention

\section{Efeitos de um Treino Breve de Meditação no Afeto Negativo, Ansiedade Traço e Atenção Concentrada}

\begin{abstract}
Resumo: A meditação tem sido associada a desfechos psicológicos positivos, mas intervenções curtas ainda são pouco investigadas. O objetivo deste ensaio piloto, randomizado e controlado, foi avaliar os efeitos de cinco dias de meditação focada no afeto positivo e negativo, na ansiedade estado e traço, bem como na atenção concentrada em uma amostra não clínica distribuída em dois grupos (experimental $=14,51,8 \%$ mulheres, $M_{\text {idade }}=23,9$; controle $=19,62 \%$ mulheres, $M_{\text {idade }}=24,9$ ). Utilizou-se a Escala de Afeto Positivo e Afeto Negativo, o Inventário de Ansiedade Traço e Estado e o Teste de Atenção Concentrada. O grupo da meditação reduziu o afeto negativo e a ansiedade traço, assim como aumentou as respostas corretas do teste de atenção concentrada, comparado ao grupo controle. Estes achados preliminares indicam que mesmo um treino breve de meditação focada pode ajudar a melhorar algumas variáveis psicológicas. Discute-se que a manifestação precoce destes benefícios pode ser especialmente relevante como uma forma de reforçar a motivação para a continuidade e regularidade da prática.
\end{abstract}

Palavras-chave: meditação, ansiedade, afeição, atenção

\section{Efectos de un Breve Entrenamiento de la Meditación en el Afecto Negativo, la Ansiedad Rasgo y la Atención Concentrada}

\begin{abstract}
Resumen: La meditación se ha relacionado con resultados psicológicos positivos, todavía intervenciones breves son poco investigadas. Este ensayo piloto controlado yaleatorio evaluó el efecto de cinco días de la meditación focalizada en el afecto positivo y negativo, en el ansiedad estado y rasgo, así como en la atención concentrada en una muestra no clínica distribuida en dos grupos (experimental $=14,51,8 \%$ mujeres, $M_{\text {edad }}=23,9$, control $=19,62 \%$ mujeres, $M_{\text {edad }}=24,9$ ). Se utilizó la Escala de Afecto Positivo y Afecto Negativo, el Inventario de Ansiedad Rasgo y Estado, y la prueba de Atención Concentrada. La meditación ha reducido el afecto negativo y el ansiedad rasgo, así como ha aumenta do las respuestas correctas de la prueba de atención concentrada, en comparación con los controles. Estos hallazgos preliminares indican que incluso un breve entrenamiento con la meditación focalizada puede ayudar a mejorar algunas variables psicológicas. Se argumenta que la manifestación temprana de estos beneficios puede ser espe-cialmente relevante como una manera de fortalecer la motivación para práctica regular.
\end{abstract}

Palabras clave: meditación, ansiedad, afección, atención

\footnotetext{
${ }^{1}$ Article derived from the doctoral dissertation of Carolina B. Menezes, under the supervision of Lisiane Bizarro, defended in 2012 in the Graduate Program in Psychology of the Universidade Federal do Rio Grande do Sul. Support: National Council for Scientific and Technological Development (CNPq - Protocol no. 140796/ 2009-6).

${ }^{2}$ Correspondence address:

Carolina B. Menezes. Curso de Psicologia, Universidade Federal de Pelotas. Av. Duque de Caxias, 250, Fragata. CEP 96030-001. Pelotas-RS, Brazil. E-mail: menezescarolina@hotmail.com
}

Meditation has been increasingly studied and integrated into the field of Psychology and health care in general, as research has indicated its contribution to outcomes associated with greater physical and mental health (Sedlmeier et al., 2012). If on the one hand continuity and regularity in its practice favor the scale of these benefits (Menezes \& Dell'Aglio, 2010; Soler et al., 2014), on the other, it is evidenced that one difficulty inherent to meditation is the potential lack of motivation for adhering to these parameters, 
which can impair the success of the anticipated results. From the behavioral point of view, it is possible to conjecture that the positive effects resulting from its practice could assist in reinforcing this behavior. As a result, it is important to assess whether brief training in meditation is capable of producing beneficial changes, bearing in mind that these could function as reinforcing stimuli for maintaining the practice.

According to systematic review and meta-analysis studies (Chiesa, Serretti, \& Jakobsen, 2013; Goyal et al., 2014; Sedlmeier et al., 2012), among the main benefits of meditation one finds the improvement of psychological variables which characterize healthier and more adaptive emotional regulation. In nonclinical samples, effect sizes were larger (medium to large) for the change of emotional variables, in particular negative ones, such as negative affect, state and trait anxiety, as well as stress; moderate (medium) for measures of attention; and weaker (small to medium) for other cognitive variables (Sedlmeier et al., 2012). For clinical samples, on the other hand, the results for stress, positive mood and attention are more inconsistent, in particular when the interventions based on meditation are compared with an active control group (Goyal et al., 2014).

Although, in these reviews, the interventions are considered to be medium and long-term, with 30 days being the shortest duration analyzed, studies evaluating short-term training - such as that lasting a few days - have also found encouraging results (Creswell, Pacilio, Lindsay, \& Brown, 2014; Ding, Tang, Tang, \& Posner, 2014; Tang et al., 2007; Zeidan, Johnson, Gordon, \& Goolkasian, 2010). It is worth emphasizing that there is no consensus in the literature in relation to the use of the terms "short-term" and "brief", as these have been used for designating different quantities of practice, such as a single session of 10 minutes (Dickenson, Berkman, Arch, \& Lieberman, 2012), five days (Tang et al., 2007), two weeks (Cavanagh et al., 2013), and experience of up to five years (Sukhsohale \& Phatak, 2012). As a result, it is emphasized that in the present article the terms "short-term" and "brief" will be used in the context of practices lasting a few days, varying between three and seven. When allusion is made to other formats of training, this will be accompanied by specification of the duration assessed.

There are only isolated studies, whose results seem to be in consonance with the meta-analysis of Sedlmeier et al. (2012), in particular regarding the effect on the emotional variables and attentional processes (Tang et al., 2007; Zeidan, Johnson, Diamond, David, \& Goolkasian, 2010; Zeidan, Johnson, Gordon, et al., 2010). Nevertheless, it is also possible to observe divergences among some of these findings. For example, two meditation training programs focusing on respiration with healthy young adults produced a significant reduction in the general rate of negative mood, compared with the control group (Tang et al., 2007; Zeidan, Johnson, Gordon, et al., 2010), but this effect was not observed in a study which found significant effect in only one component of negative mood, namely, fatigue (Zeidan, Johnson, Diamond, et al., 2010). In relation to anxiety, two studies with a nonclinical sample of young adults found greater reduction of state anxiety after finishing an intervention with respiration meditation, in relation to the control condition (Zeidan, Johnson, Diamond, et al., 2010; Zeidan, Johnson, Gordon, et al., 2010), but in only one of these (Zeidan, Johnson, Gordon, et al., 2010), as well as in a separate study (Zeidan, Gordon, Merchant, \& Goolkasian, 2010), did the state anxiety reduce significantly only at the end of each meditation session. Another study found that, compared with the control group, the university students of one nonclinical sample who participated in an intervention with mindfulness meditation presented greater reduction of symptoms of anxiety, it being the case that this benefit was greater for the participants who had reported moderate baseline levels (Chen, Yang, Wang, \& Zhang, 2013). Moreover, one shortterm intervention termed Integrative Body-Mind Training (IBMT) also produced improvements in the reporting of positive affect and negative affect of healthy university students (Dinget al., 2014).

Other findings derive from studies in which emotional responses were assessed experimentally through biological parameters. For example, it was demonstrated that only those people (healthy university students) who participated in an IBMT intervention - rather than relaxation - significantly reduced the levels of cortisol which had increased through stress induction with arithmetic (Tang et al., 2007). However, during the induction of stress through social evaluation, significant differences were not found in the levels of cortisol and blood pressure in the group of healthy adults who undertook mindfulness meditation, although the meditators did report a lesser perception of stress in relation to the stressor situation (Creswell et al., 2014). It is important to highlight that these divergent results derived from distinct protocols. For example, Tang et al. (2007) used cortisol as a parameter of recovery from the stress response, as this was assessed in the period following the induction. In the study by Creswell et al. (2014), on the other hand, cortisol was measured only during the induction of stress.

Arterial pressure also did not show significant changes during a normal post-test evaluation (without induction of stress), the objective of which was to measure the effects of brief training on autonomic variables (Zeidan, Johnson, Gordon, et al., 2010). However, this (Zeidan, Johnson, Gordon, et al., 2010) and another study (Tang et al., 2009) showed that cardiac frequency was significantly lower in the post-test in the group which participated in training in meditation, in comparison with the control groups.

A smaller quantity of studies also investigated the effect of short training programs on some attentional processes. A few days' practice seems to have positively influenced executive attention, measured with a conflict resolution task (Tang et al., 2007), as well as sustained attention, such as indexed by working memory tasks (Zeidan, Johnson, Diamond, et al., 2010). Furthermore, two studies showed an increase in the scores for dispositional mindfulness, obtained through the Freiburg Mindfulness Inventory, whose construct means the experience of being alert and aware regarding the present moment (Zeidan, Gordon, et al., 2010; Zeidan, Johnson, Diamond, et al., 2010). 
Many of these studies evaluated training programs using mindfulness meditation, which emphasizes the awareness of the present moment through the maintenance of a state of alertness and vigilance which must be accompanied by an experience of acceptance and nonjudgment (Chen et al., 2013; Creswell et al., 2014; Zeidan, Johnson, Diamond, et al., 2010; Zeidan, Johnson, Gordon, et al., 2010). Others tested an intervention termed Integrative Mind-Body Training (IMBT; Dinget al., 2014; Tang et al., 2007), which can also be simply termed integrative meditation. This training combines practices of bodily relaxation, respiration, guided imagination and mindfulness meditation, all accompanied by background music, seeking to facilitate the occurrence, without effort, of a greater control and greater awareness of internal processes.

This study's objective was to evaluate the effects of five days of focused meditation (also known as concentrative) on positive and negative affect, on state anxiety and trait anxiety, as well as on concentrated attention, in a nonclinical sample distributed in two groups: focused meditation and waiting list control group. Regarding positive and negative affect (for a review of the construct, see Giacomoni, 2004), it was sought to assess whether focused meditation can be equally capable of contributing to the increase in the positive affect and reduction of the negative affect. In the focused meditation technique, the practitioners train sustained and concentrated attention on a single focus in a more systematic way, so as to develop the skill of more easily not becoming engaged, or of disengaging oneself from the usual mental processes, which are often automatized and dysfunctional (Lutz, Slagter, Dunne, \& Davidson, 2008). This meditation has already been tested in one study, evaluating a six-week intervention, whose results showed a reduction in the self-reporting of negative affect and of trait anxiety, and an improvement in the performance of attention in healthy university students (Menezes et al., 2013).

\section{Method}

\section{Participants}

This was a pilot study, undertaken through a randomized and controlled trial with university students of the Federal University of Rio Grande do Sul (UFRGS), who were invited to participate in the study via email and posters placed in the University's three campuses. A total of 120 students showed interest in participating. Of these, the following were excluded: those who were not aged between 20 and 40 years old; who did not have normal or corrected vision; who reported some psychiatric or neurological disorder; who reported the use of psychoactive medication; who were undergoing psychotherapeutic treatment; and/ or who reported previous experience with meditation or yoga. Initially, 46 eligible participants were randomly and equally distributed in two groups (focused meditation (FM), and waiting list control group (CG), but the final sample encompassed 33 participants [FM: $n=14,51.8 \%$ women, $100 \%$ single, $M_{\text {age }}=23.9$ years $(S D=4.45)$; CG: $n=19,62 \%$ women, $88 \%$ single, $\left.M_{\text {age }}=24.9(S D=5.1)\right]$.
These variables did not differ between groups ( $p \geq .05)$, and neither was there a significant difference in the attrition rate $\left[\chi^{2}(1)=3.4, p=.09\right]$. All the losses in the meditation group occurred because the participants stopped attending the training sessions. In the control group, on the other hand, all the losses represent participants who did not attend the second evaluation. In spite of there being no difference in the attrition rate, it is worth emphasizing that throughout the week of the meditation training, the weather conditions were characterized by intense cold and heavy rain, as expected for July in Southern Brazil. It is believed, therefore, that this may be a possible explanation for the greater loss observed in the meditation group. Supporting this supposition, of the 19 participants of the control group who later received this intervention, only one desisted from the training.

\section{Instruments}

Sociodemographic questionnaire. Developed for the present study with the aim of investigating sociodemographic variables, as well as the other variables referent to the inclusion and exclusion criteria.

Self-Report Questionnaire - SRQ (Mari \& Williams, 1986). The SRQ consists of 20 questions investigating the presence of minor psychiatric symptoms, and in three questions investigating the presence of psychotic symptoms through binary responses of yes or no. The new version validated in Brazil (Mari \& Williams, 1986) presented coefficients of sensitivity and specificity of $83 \%$ and $80 \%$, respectively. So as to exclude participants who could potentially meet the criteria for some psychiatric diagnoses, the cut-off points adopted for men and women were, respectively, seven and six positive responses.

Adult Self-Report Scale - ASRS (Mattos et al., 2006). The ASRS consists of 18 items covering attention deficit hyperactivity disorder (ADHD) in adult life. The responses are provided on a five-point Likert scale $(0=$ never, $1=$ rarely, $2=$ sometimes, $3=$ frequently, $4=$ very frequently). Positive responses include frequently and very frequently, and sometimes for some questions (items 3, 4, 5 and 9 for part A; items 2, 7, and 9 for part B). The cut-off point for a possible diagnosis includes a minimum of six symptoms in at least one domain (inattention: items 1 - 9 in part A; hyperactivity: items 1 - 9 in part B), or in both. A score above 24 is considered highly suggestive of diagnosis. As this is a scale which seeks to viabilize the diagnosis of ADHD through a list of symptoms in accordance with the criteria of the DSM-IV, its evaluation parameters cover the rates of sensitivity (56.3\%), specificity $(98.3 \%)$ and accuracy of classification (96.2\%), all being satisfactory. This scale was used both in the tracking, in order to exclude possible cases of ADHD, as also in the pre-test, in order to compare the number of symptoms between the groups in the baseline. In the event of there being some difference between the groups, this variable would be controlled in the analyses of the test of attention.

State-Trait Anxiety Inventory - IDATE (Pasquali, Pinelli Júnior, \& Solha, 1994). This inventory is made up of two 
scales, each one with 20 questions which separately measure state anxiety and trait anxiety. In the state anxiety, the questions refer to how the person feels at the time the assessment is being undertaken; the questions for the trait anxiety represent how the person normally feels. The responses indicate the levels of anxiety in these two contexts through the use of a four-point Likert scale varying from not at all to a lot. The higher the score, the higher the level of anxiety. The Brazilian version presents Cronbach alphas of .89 for the scale of state anxiety and .88 for trait anxiety (Pasquali et al., 1994).

The Concentrated Attention Test - AC (Cambraia, 2003). The AC is a Brazilian psychometric test which assesses concentrated attention. Its test-retest coefficient is .73 . Using pencil or pen and a response sheet, the participants have up to five minutes to mark the highest number possible of previously-established formats of triangle, which are distributed randomly amongst other types of triangles. This test's evaluation includes the rates of correct responses, errors, omissions, and total score.

The Positive and Negative Affect Scale - PANAS (Zanon, Bastianello, Pacico, \& Hutz, 2013). This scale, adapted for Brazil, consists of 20 descriptors for each category of affect: positive (e.g.: enthusiastic; $\alpha=.88$ ) and negative (e.g.: fearful; $\alpha=.86$ ). Using a five-point Likert scale varying between very little and extremely, the participants must evaluate the degree to which they have recently experienced these affects. The scores for positive affect and negative affect are created separately, it being the case that the higher the score, the greater the report of the affect.

Assessment of the Training. In the posttest, the participants evaluated how satisfied they were with the training $(1=$ not at all satisfied, $2=$ little satisfied, $3=$ indifferent, $4=$ satisfied, $5=$ very satisfied $)$ and how useful they had found the techniques $(1=$ not at all useful, $2=$ little useful, $3=$ indifferent, $4=$ useful, $5=$ very useful).

\section{Procedure}

Data collection. After the publicizing of the study, those volunteers interested in participating answered the tracking questionnaires in an online format using the Survey Monkey platform. The eligible participants were randomly distributed to one of the groups and were then contacted for arranging the visit to the laboratory for the pre-test evaluation. The two evaluations (pre-test and post-test) took place during the two weeks which preceded and followed the intervention, respectively, in the Institute of Psychology, Experimental Psychology, Neuroscience and Behavior Laboratory (UFRGS). The meditation training included five consecutive daily meetings which lasted approximately 90 minutes. The participants were instructed regarding the importance of participating in all five days, as missing one or more meetings would result in their exclusion from the study. The participants in the control group had no activity during this period, but received the same training at the end of the study. Training was conducted by the first author, a psychologist with experience in group work and 11 years of practice in yoga and meditation. The meetings had a structure which always proceeded in the following order of activities: group conversation involving doubts, difficulties and reports of experiences; information and instructions for the practices; diaphragmatic breathing exercises; formal practice; and closing, with more time for conversations and exchanging experiences. In the first two meetings, the formal practice lasted 15 and 20 minutes, respectively. In the remaining three meetings, the practice lasted 30 minutes. The participants could sit cross-legged on a mat, or on a chair without resting their backs on it, and with their feet firmly supported on the ground. Regardless of whether they were on the ground or on a chair, the participants could change their seating option when they wanted to. In the event of discomfort, the participants could move to make their body more comfortable, but were instructed to be discreet and silent so as not to inconvenience the others. In the breathing exercises, all were instructed to pay attention to the respiration and to the abdominal movements which accompany both inspiration and exhalation, trying to lightly prolong exhalation. In the meditation, it was emphasized that no particular effort should be made for breathing. However, so as to characterize the focused attention meditation, as well as to make it possible for the participants to maintain the focus of their attention on breathing, the same were instructed to mentally count each exhalation (it is noted that other types of focus, such as mantras, were not used, in order to avoid any link with philosophical or spiritual traditions). In the first three days, the counting covered cycles from one to ten, and in the last two days, the participants undertook a regressive count from 100 to one (always with one number per exhalation). In both counts, the participants were instructed to restart the cycle from the beginning (number one, or number 100), if they perceived that they had been distracted or had lost count. They were not requested to undertake additional practices in the periods between each meeting.

Data analysis. In the pre-test, a one-way ANOVA was used to compare each one of the outcomes, separately, between the groups. In the post-test, the General Linear Model (GLM) for repeated measures was used to compare each one of the outcomes separately, taking into account the factors of group (FM $v s \mathrm{CG}$ ) and time (pre-test $v s$ post-test). The ANOVA and polynomial contrasts were later used when necessary. In the post-test, the Pearson Partial Correlation test was also used, to evaluate the relationship between the outcomes within each group, controlling the scores of each outcome in the pre-test. For all the analyses, a level of significance of 0.05 was adopted, and SPSS version 20.0 was used.

\section{Ethical Considerations}

This study was approved by the Research Ethics Committee of the Institute of Psychology at the Universidade Federal do Rio Grande do Sul, under registration number 25000.089325/2006-58. All the participants provided informed consent prior to the assessments. 


\section{Results}

\section{Pre-Test}

There was no significant difference for any outcome between the groups in the pre-test ( $p \geq .05$; means and standard deviations can be observed in Table 1). In addition to this, the groups did not differ in relation to the ASRS score $[F(1,44)=$ $-.24, p=.80]$ and, for this reason, it was not necessary to control this variable in the subsequent analyses of the AC.

\section{Post-Test}

Both the sociodemographic variables and the data regarding the outcomes of the pre-test were compared among the participants who completed the study and the participants who dropped out. No significant difference was found $(p>$ $.05)$. All the participants of the meditation group evaluated the techniques as useful ( $40 \%)$ and very useful (60\%), there being no responses for not useful at all or little useful. In the same way, all reported medium $(40 \%)$ and high satisfaction $(60 \%)$ with the training, there being no responses of dissatisfaction or neutral responses.

Table 1 shows descriptive data for all the outcomes evaluated. In relation to the positive affect, there was a main effect for time $\left[F(1,31)=4.39, p=.04, \eta^{2}=.12\right]$, but no interaction between group $v s$ time $[F(1,31)=.62, p=.43$, $\left.\eta^{2}=.02\right]$. For the negative affect, there was both a main effect for time $\left[F(1,31)=37.7, p<.001, \eta^{2}=.54\right]$, with an interaction for group $\mathrm{v}$ time $\left[F(1,31)=4.88, p=.03, \eta^{2}=\right.$ .14]. In the meditation group, the reduction of the negative affect between the pre- and post-test was greater $[F(1,13)$ $\left.=41.65, p<.001, \eta^{2}=.76\right]$, in comparison with the control group $\left[F(1,18)=7.60, p=.01, \eta^{2}=.29\right]$.

In relation to the state anxiety, there was a main effect for time $\left[F(1,31)=5.61, p=.02, \eta^{2}=.15\right]$, without any interaction between group vs time $[F(1,31)=1.31, p=.26$, $\left.\eta^{2}=.04\right]$. For the trait anxiety, there was main effect for time $\left[F(1,31)=4.90, p=.03, \eta^{2}=.13\right]$, as well as interaction between group vs time $\left[F(1,31)=4.05, p=.04, \eta^{2}=.12\right]$. The meditation group presented a significantly greater reduction of the trait anxiety trait between the pre- and the post-test $\left[F(1,13)=6.01, p=.02, \eta^{2}=.31\right]$, in comparison with the control group $\left[F(1,18)=0.3, p=.86, \eta^{2}=.00\right]$.

Referent to the test of concentrated attention, there was a main effect for time for the rate of correct answers $[F(1,31)$ $\left.=13.30, p=.001, \eta^{2}=.30\right]$, as well as an interaction between group vs time for this rate $\left[F(1,31)=4.21, p=.05, \eta^{2}=\right.$ .10]. The meditation group presented a significantly greater increase of correct responses between the pre- and the posttest $\left[F(1,13)=14.77, p=.002, \eta^{2}=.53\right]$, in comparison with the control group $\left[F(1,18)=1.9, p=.18, \eta^{2}=.09\right]$. Significant responses were not found for the other rates of the $\mathrm{AC}$ (error, omission or total score: $p>.05$ ).

\section{Correlations}

Only in the meditation group was there significant correlation between the scores of post-test negative affect and correct responses of the $\mathrm{AC}$, even controlling for the scores of the pre-test for these outcomes (FM: $r=-.63, p=.02$; CG: $r=$ $-.08, p=.73)$.

Table 1

General Linear Model (GLM) for Repeated Measures: Interaction of the Outcomes Between Time (Pre-vs. Post-Test) and Group (Meditation vs. Control); Single-Factor ANOVA: Post-Hoc Comparison of the Outcomes (Mean and Standard Deviation) Between Pre- and Post-Test for Each Group

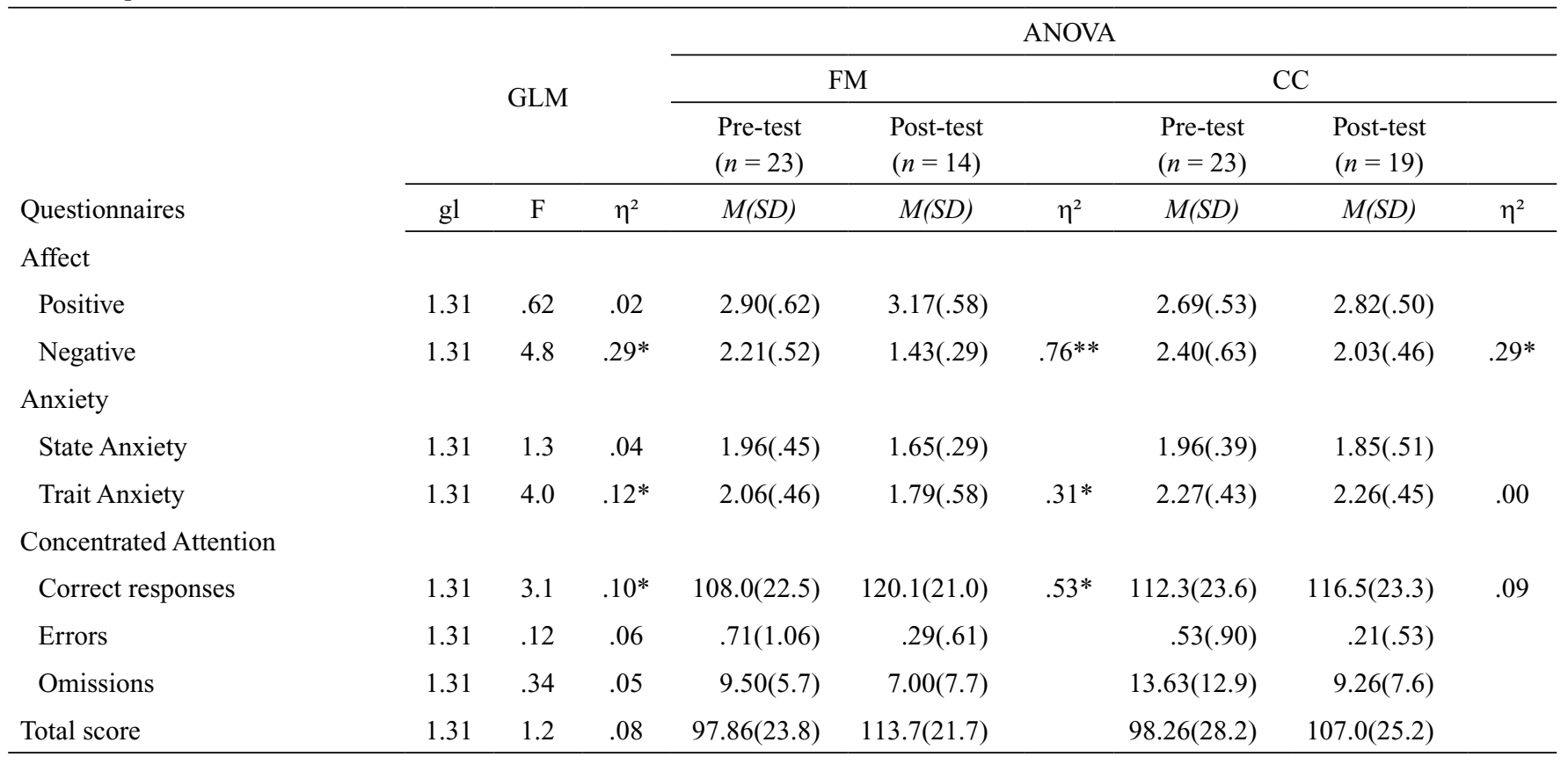

Note. GLM = General Linear Model for repeated measures; ANOVA = single-factor variance analysis; FM = focused meditation; CG = waiting list control group.

${ }^{*} p \leq .05 . * * p<.001$. 
The same pattern was observed for the correlation of the scores of post-test negative affect and trait anxiety, in which the scores of the pre-test for these outcomes were also controlled (FM: $r=.86, p=<.001$; CG: $r=.36, p=.15$ ).

\section{Discussion}

This pilot study investigated the effect of brief training of focused meditation, in which practices with a mean duration of 20 minutes were undertaken for five consecutive days, on the outcomes of affect, anxiety and concentrated attention in a nonclinical sample. The results showed that in comparison with a waiting list control group, the meditation training produced significant and positive changes, in particular the reduction of the self-reported scores for negative affect and trait anxiety, as well as better performance in the concentrated attention tests, as indicated by the greater rate of correct responses. There was also an inverse relationship between the scores of post-test negative affect and the correct responses in the concentrated attention test, and a positive relationship between the scores of post-test negative affect and trait anxiety. Furthermore, all the participants evaluated the techniques as useful or very useful (nobody responded not at all useful, a little useful or little use), and all reported medium or high satisfaction with the training, there being no dissatisfied or neutral responses. These findings support other studies which also evaluated emotional and attentional variables following short interventions varying between three and seven days (Chen et al., 2013; Ding et al., 2014; Tang et al., 2007; Zeidan, Johnson, Diamond, et al., 2010; Zeidan, Johnson, Gordon, et al., 2010). The results also corroborate a study which evaluated a six-week version of the same training of focused meditation, particularly in relation to the fact that in both interventions there was a reduction in the negative affect, although no significant change was observed in the positive affect, as well as reduction of trait anxiety (Menezes et al., 2013). In addition to this, the results found complement the literature regarding short training programs, demonstrating that positive effects can also occur through an intervention using focused meditation.

There are discussions and evidence that concentrative meditation (also called focused attention) and mindfulness meditation (also described as open monitoring meditation) can involve some specific and distinct processes and mechanisms (Lutz et al., 2008; Tang \& Posner, 2013). For example, there is the proposition that concentrative meditation is more related to the increase in mental stability, while practices of mindfulness are more associated with the production of insights regarding the nature of mental processes (Lutz et al., 2008). However, from a psychological point of view, either cognitive, emotional, social or behavioral, different modes of practice seem to generate equivalent changes, such as the reduction of negative emotions and an increase in attentional capacity (Sedlmeier et al., 2012). In addition to this, the variable "type of meditation" did not moderate the superior effect that experienced practitioners had regarding dispositional mindfulness scores, in comparison with non-practitioners (Soler et al., 2014). In a phenomenological perspective, therefore, it is suggested that different types of meditation, in particular sitting and silent, share a common objective, namely, to learn to develop greater awareness of mental patterns, without experiencing the reactions that these would normally evoke. For this reason, the use of an anchor (e.g. breathing) is usually used, both for helping to stabilize the mind, and to allow a broader awareness that mental activity is constantly characterized by fluctuations. As a result, through the exercise of always returning awareness to the present moment, one improves the capacity to disengage attention from the process of automatically associating and reacting to thoughts, emotions, feelings and other internal experiences.

Regardless of the type of meditation, one variable which seems to favor the skills developed with practice is its duration, in particular time (in months or years) and weekly frequency (Menezes \& Dell'Aglio, 2010; Soler et al., 2014). In consonance with these findings, it has been observed that some patterns of responses, such as the control of the processing of distracting stimuli, differ when beginners and experienced persons are compared, both in relation to the attentional domain and to the emotional domain (Brefczynski-Lewis, Lutz, Schaefer, Levinson, \& Davidson, 2007). Nevertheless, there are data which help one to consider why a five days training is already able to produce positive changes, such as those observed in the present study, namely, reduced negative affect and trait anxiety, as well as improved concentrated attention.

One hypothesis is the possible increase in dispositional mindfulness, considered a type of mental activity which is less reflexive and conditioned, and which involves greater attention to, and awareness of, the present moment, as well as greater acceptance (Brown \& Ryan, 2003). It was demonstrated that a few days of training in meditation produced an increase in the scores for dispositional mindfulness, and that these scores were negatively correlated with the scores for state anxiety (Zeidan, Gordon, et al., 2010). In addition to this, people with a lower score for dispositional mindfulness had greater reactivity to a stressful situation that was induced experimentally (Creswell et al., 2014). It seems, therefore, that this mental quality may help to lower reactivity to negative stimuli. Indeed, "non-reactivity" encompasses one of the facets which make up the construct of mindfulness in a multifactorial perspective. Moreover, "non-reactivity" and "observation" were the facets which presented the difference with the greatest effect size between practitioners experienced in meditation and non-practitioners (Soler et al., 2014).

In this same direction, it is suggested that the possible increase of mindfulness could also help in reducing the bias for negative stimuli, reducing a process which privileges negative emotions. In consonance with the reduction of negative affect in the present study, people who undertook a single 15-minute session of meditation prior to a verbal learning test - with words that had negative, positive and neutral valence - remembered a significantly lower quantity of negative words in the delayed recall task, compared with a control group which did not undertake meditation (Alberts $\&$ Thewissen, 2011). As a result, based on the proposition of a common objective underlying meditation practices, it is 
possible to speculate that an increase in mindfulness may be related to a better attentional regulation, helping in the skill of avoiding the engagement of mental resources in negative stimuli, or facilitating disengagement from this class of stimuli. Supporting this proposition, it was observed that the greater recruiting of the areas involved in attentional processes - the parietal and pre-frontal structures - in people who had been meditating for the first time for 10 minutes, compared to people who had left their mental activity free, was moderated by a greater level of dispositional mindfulness (Dickenson et al., 2012). Despite not having specific data for mindfulness, the present study found an improvement of concentrated attention after the five-day training, and this was significantly correlated with the lower reporting of negative affect in the meditation group.

However, it is known that bias for perception and processing of negative stimuli can be significantly influenced by high levels of anxiety (Bishop, 2007). In the present study there was a significant correlation between lower trait anxiety and reduced negative affect only in the meditation group. Therefore, bearing in mind that dispositional mindfulness is related both to attention (Dickenson et al., 2012) and anxiety (Zeidan, Gordon, et al., 2010), and considering that dispositional mindfulness was not an outcome directly measured in this study, it is important that future investigations aim to test mediational models with these variables. The possible influence of increased dispositional mindfulness, as well as of better attentional regulation in reducing bias for negative stimuli may be mediated or moderated by the effect of anxiety; alternatively, these variables may produce additive effects on the bias.

Another potential explanation for the benefits of brief training is the idea that even with only a few days, meditation helps to reduce the harmful effect of the depletion of cognitive resources. For example, this relationship was investigated in an experimental context in which a selfcontrol task was to be undertaken by two distinct groups, one which had previously undergone induction of depletion of resources with an emotional suppression task (that is, a task which also required self-control), and another which had not undergone the depletion. This study had two main findings. In a general sample, unrelated to meditation, the people who suffered depletion of resources had significantly worse performance in the subsequent self-control task. In another sample made up of people who had learned meditation for three days, the people who undertook a meditation session after undergoing depletion performed in the subsequent task as well as the participants from the general sample who had not undergone depletion (Friese, Messner, \& Schaffner, 2012). One hypothesis, therefore, is that meditation helps to restore these resources, or to minimize the harm that results from the depletion.

Although the initial stages of meditative practice require more effort and greater use of mental resources (Brefczynski-Lewis et al., 2007; Kozasa et al., 2012), it is known that the attentional cognitive pattern underlying the practice of meditation is distinct from the pattern observed in analytical cognitive tasks, such as self-referential processes
(Farb et al., 2007). This distinction supports experimental cognitive investigations, which demonstrate an inverse pattern of susceptibility to distraction for conditions of high attentional demand and high cognitive demand (Lavie, 2005). In particular, higher attentional demand prevents the interference of distractors, while the opposite is observed when there is a high cognitive load, such as a major demand on working memory. Therefore, even though the beginning of training in meditation may be associated with greater activation of some cerebral areas, in comparison with practitioners with greater experience (Brefczynski-Lewis et al., 2007; Kozasa et al., 2012), it is proposed that this type of practice involves a mechanism which opposes the depletion of resources, and can function as a tool for preventing or reducing the harmful effect of the depletion deriving from other activities. In conformity with this propositions, there is evidence that depletion of cognitive resources is significantly related to greater negative affect and fatigue (Hagger, Wood, Stiff, \& Chatzisarantis, 2010), whose outcomes presented improvement after different brief training programs in meditation (Ding et al., 2014; Tang et al., 2007; Zeidan, Johnson, Diamond, et al., 2010; Zeidan, Johnson, Gordon, et al., 2010).

Finally, it is also possible to suggest that at the base of all the hypotheses discussed, one can find the effects of neurophysiological mechanisms. For example, some short training programs produced better regulation of specific autonomic parameters, such as lower cardiac frequency and skin conductance (Tang et al., 2009; Zeidan, Johnson, Gordon, et al., 2010). The fact that these rates were measured in normal conditions of evaluation (comparing pre- and post-test), and not as an immediate response to a session of meditation, is suggestive that the findings represent a change in the basal level of functioning. From this perspective, these findings help to explain the effect which we observed in trait anxiety, as this evaluates the levels of anxiety in relation to general functioning, and not at a single, specific point. In addition to this, the greater recruitment of attentional circuits during a single 10 minutes meditation session -focused breathing - relative to a free mental activity (Dickenson et al., 2012), indicates that even the initial stages of practice have the potential to stimulate the plasticity of neural mechanisms underlying attention. As a result, it is suggested that this plasticity underlies the relationship between meditation and better attentional regulation and helps to explain why better attentional regulation can be generalized to other activities and contexts.

In sum, this study presents preliminary data that a training program of five consecutive days, of approximately 20 minutes of focused meditation, was able to generate improvement in some psychological variables, in particular negative affect, trait anxiety and concentrated attention, in a non-clinical sample of university students. As a pilot study, this work presents some important methodological limitations, such as its small sample size, high drop-out rate, the lack of an active control group, and the non-evaluation of the individual effects of the intervention. In addition to this, the sample of healthy university students limits the external validity of the findings. However, it stands out 
that the changes found presented moderate effect sizes, supporting the internal validity of the results, as well as the fact that the techniques employed were evaluated as useful and satisfactory by the participants.

The study also did not use a psychometric measure of mindfulness, and neither did it evaluate neurophysiological responses, such that some hypotheses proposed and discussed must be considered with caution. Furthermore, one limitation of this and of all the studies cited which investigated the effect of brief meditation trainings is the absence of follow-up, not allowing inferences to be made regarding the permanence or durability of the changes observed. However, in relation to this limitation, it is highlighted that the relevance for evaluating the effect of a short-term training program is not owed to the possibility of inferring that a few days of practice can generate sufficient and long-lasting effects. The main rationale is to be able to evaluate whether some benefit can be manifested within few days of training, as early effects could function as motivators, facilitating the maintenance and regularity of the practice, and, consequently, the maintenance of the psychological benefits.

It is concluded that the changes observed indicate that meditation trainings have important potential for applicability, justifying its incorporation into different contexts, for example, other educational or work environments, services of mental and physical healthcare, and even in the context of individual interventions, either in the public or private sectors. Finally, it is hoped that this pilot study may contribute to fostering the implementation of such interventions into continuous programs of socio-emotional support offered in the university context.

\section{References}

Alberts, H. J. E. M., \& Thewissen, R. (2011).The effect of a brief mindfulness intervention on memory for positively and negatively valenced stimuli. Mindfulness, 2(2), 73-77. doi:10.1007/s12671-011-0044-7

Bishop, S. J. (2007). Neurocognitive mechanisms of anxiety: An integrative account. Trends in Cognitive Sciences, 11(7), 307-316. doi:10.1016/j. tics.2007.05.008

Brefczynski-Lewis, J. A., Lutz, A., Schaefer, H. S., Levinson, D. B., \& Davidson, R. J. (2007). Neural correlates of attentional expertise in long-term meditation practitioners. Proceedings of the National Academy of Sciences of the United States of America, 104(27), 11483-11488. doi:10.1073/pnas.0606552104

Brown, K. W., \& Ryan, R. M. (2003). The benefits of being present: Mindfulness and its role in psychological wellbeing. Journal of Personality and Social Psychology, 84(4), 822-848. doi:10.1037/0022-3514.84.4.822

Cambraia, S. V. (2003). Teste AC: Manual [AC test: Guideline] (Vol. 3). São Paulo, SP: Vetor.

Cavanagh, K., Strauss, C., Cicconi, F., Griffiths, N., Wyper, A., \& Jones, F. (2013). A randomised controlled trial of a brief online mindfulness-based intervention.
Behaviour Research and Therapy, 51(9), 573-578. doi:10.1016/j.brat.2013.06.003

Chen, Y., Yang, X., Wang, L., \& Zhang, X. (2013). A randomized controlled trial of the effects of brief mindfulness meditation on anxiety symptoms and systolic blood pressure in Chinese nursing students. Nurse Education Today, 33(10), 1166-1172. doi:10.1016/j. nedt.2012.11.014

Chiesa, A., Serretti, A., \&Jakobsen, J. C. (2013). Mindfulness: Top-down or bottom-up emotion regulation strategy? Clinical Psychology Review, 33(1), 82-96.

Creswell, J. D., Pacilio, L. E., Lindsay, E. K., \& Brown, K. W. (2014). Brief mindfulness meditation training alters psychological and neuroendocrine responses to social evaluative stress. Psychoneuroendocrinology, 44, 1-12. doi:10.1016/j.psyneuen.2014.02.007

Dickenson, J., Berkman, E. T., Arch, J., \& Lieberman, M. D. (2012). Neural correlates of focused attention during a brief mindfulness induction. Social Cognitive and Affective Neuroscience,8(1), 40-47. doi:10.1093/scan/ nss 030

Ding, X., Tang, Y.-Y., Tang, R., \& Posner, M. I. (2014). Improving creativity performance by short-term meditation. Behavioral and Brain Functions: BBF, 10, 9. doi:10.1186/1744-9081-10-9

Farb, N. A. S., Segal, Z. V., Mayberg, H., Bean, J., McKeon, D., Fatima, Z., \& Anderson, A. K. (2007). Attending to the present: Mindfulness meditation reveals distinct neural modes of self-reference. Social Cognitive and Affective Neuroscience, 2(4), 313-322. doi:10.1093/scan/nsm030

Friese, M., Messner, C., \& Schaffner, Y. (2012). Mindfulness meditation counteracts self-control depletion. Consciousness and Cognition, 21(2), 1016-1022. doi:10.1016/j.concog.2012.01.008

Giacomoni, C. H. (2004). Bem-estar subjetivo: Em busca da qualidade de vida [Subjective well-being: The search for quality of life]. Temas em Psicologia, 12(1), 43-50.

Goyal, M., Singh, S., Sibinga, E. M., Gould, N. F., RowlandSeymour, A., Sharma, R., . . . Haythornthwaite, J. A. (2014). Meditation programs for psychological stress and well-being: A systematic review and meta-analysis. JAMA Internal Medicine, 174(3), 357-368. doi:10.1001/ jamainternmed.2013.13018

Hagger, M. S., Wood, C., Stiff, C., \& Chatzisarantis, N. L. D. (2010). Ego depletion and the strength model of selfcontrol: A meta-analysis. Psychological Bulletin, 136(4), 495-525. doi:10.1037/a0019486

Kozasa, E. H., Sato, J. R., Lacerda, S. S., Barreiros, M. A. M., Radvany, J., Russell, T. A., . . A Amaro, E., Jr. (2012). Meditation training increases brain efficiency in an attention task. NeuroImage, 59(1), 745-749. doi:10.1016/j.neuroimage.2011.06.088

Lavie, N. (2005). Distracted and confused?: Selective attention under load. Trends in Cognitive Sciences, 9(2), 75-82. doi:10.1016/j.tics.2004.12.004

Lutz, A., Slagter, H. A., Dunne, J. D., \& Davidson, R. J. (2008). 
Attention regulation and monitoring in meditation. Trends in Cognitive Sciences, 12(4), 163-169. doi:10.1016/j. tics.2008.01.005

Mari, J. J., \& Williams, P. (1986). A validity study of a psychiatric screening questionnaire (SRQ-20) in primary care in the city of São Paulo. British Journal of Psychiatry, 148(1), 23-26. doi:10.1192/bjp.148.1.23

Mattos, P., Segenreich, D., Saboya, E., Louzã, M., Dias, G., \& Romano, M. (2006). Adaptação transcultural para o português da escala Adult Self-Report Scale para avaliação do transtorno de déficit de atenção e hiperatividade (TDAH) em adultos [Transcultural adaptation of the Adult Self-Report Scale into portuguese for evaluation of adult attention-deficit/hyperactivity disorder (ADHD)]. Revista de Psiquiatria Clínica, 33(4), 188-194. doi:10.1590/S0101-60832006000400004

Menezes, C. B., Couto, M. C. P., Buratto, L. G., Erthal, F., Pereira, M. G., \& Bizarro, L. (2013). The improvement of emotion and attention regulation after a 6-week training of focused meditation: A randomized controlled trial. Evidence-Based Complementary and Alternative Medicine, 2013, 984678. doi:10.1155/2013/984678

Menezes, C. B., \& Dell'Aglio, D. D. (2010). The relationship between the practice of sitting and silent meditation, and psychological well-being and the effects of personality traits. Revista Interamericana de Psicología, 44(1), 140149.

Pasquali, L., Pinelli Júnior, B., \& Solha, A. C. (1994). Contribuição à validação e normatização da escala de ansiedade traço-estado do IDATE [Contributions to the validation and normalization of the state-trait anxiety inventory - STAI]. Psicologia: Teoria e Pesquisa, 10(3), 411-420.

Sedlmeier, P., Eberth, J., Schwarz, M., Zimmermann, D., Haarig, F., Jaeger, S., \& Kunze, S. (2012). The psychological effects of meditation: A meta-analysis. Psychological Bulletin, 138(6), 1139-1171. doi:10.1037/ a0028168

Soler, J., Cebolla, A., Feliu-Soler, A., Demarzo, M. M. P., Pascual, J. C., Baños, R., \& García-Campayo, J. (2014). Relationship between meditative practice and self-reported mindfulness: The MINDSENS composite index. PLoSOne, 9(1), e86622. doi:10.1371/journal. pone. 0086622

Sukhsohale, N. D., \& Phatak, M. S. (2012). Effect of shortterm and long-term Brahmakumaris Raja Yoga meditation on physiological variables. Indian Journal of Physiology and Pharmacology, 56(4), 388-392. Retrieved from http://www.ijpp.com/index.php

Tang, Y.-Y., Ma, Y., Wang, J., Fan, Y., Feng, S., Lu, Q., . . . Posner, M. I. (2007). Short-term meditation training improves attention and self-regulation. Proceedings of the National Academy of Sciences of the United States of America, 104(43), 17152-17156. doi:10.1073/ pnas.0707678104

Tang, Y.-Y., Ma, Y., Fan, Y., Feng, H., Wang, J., Feng, S., .. . Fan, M. (2009). Central and autonomic nervous system interaction is altered by short-term meditation. Proceedings of the National Academy of Sciences of the United States of America, 106(22), 8865-70. doi:10.1073/ pnas.0904031106

Tang, Y.-Y., \& Posner, M. I. (2013). Tools of the trade: Theory and method in mindfulness neuroscience. Social Cognitive and Affective Neuroscience, 8(1), 118-120. doi: $10.1093 / \mathrm{scan} / \mathrm{nss} 112$

Zanon, C., Bastianello, M. R., Pacico, J. C., \& Hutz, C. S. (2013). Desenvolvimento e validação de uma escala de afetos positivos e negativos [Development and validation of a positive and negative affect scale]. Psico-USF, 18(2), 193-201. doi:10.1590/S1413-82712013000200003

Zeidan, F., Gordon, N. S., Merchant, J., \& Goolkasian, P. (2010). The effects of brief mindfulness meditation training on experimentally induced pain. The Journal of Pain: Official Journal of the American Pain Society, 11(3), 199-209. doi:10.1016/j.jpain.2009.07.015

Zeidan, F., Johnson, S. K., Diamond, B. J., David, Z., \& Goolkasian, P. (2010). Mindfulness meditation improves cognition: Evidence of brief mental training. Consciousness and Cognition, 19(2), 597-605. doi:10.1016/j.concog.2010.03.014

Zeidan, F., Johnson, S. K., Gordon, N. S., \& Goolkasian, P. (2010). Effects of brief and sham mindfulness meditation on mood and cardiovascular variables. The Journal of Alternative and Complementary Medicine, 16(8), 867873. doi:10.1089/acm.2009.0321

Carolina Baptista Menezes is a Ph.D. in Psychology from Universidade Federal do Rio Grande do Sul and a Professor at Universidade Federal de Pelotas.

Lisiane Bizarro is an Associate Professor of the Universidade Federal do Rio Grande do Sul.

Received: Aug. 18, 2014

1st Revision: Jan. 7, 2015

Approved: Jan. 27, 2015

How to cite this article:

Menezes, C. B., \& Bizarro, L. (2015). Effects of a brief meditation training on negative affect, trait anxiety and concentrated attention. Paidéia (Ribeirão Preto), 25(62), 393-401. doi:10.1590/1982-43272562201513 